\title{
Improving the accuracy of cosmic magnification statistics
}

\author{
B. Ménard ${ }^{1,2}$, T. Hamana ${ }^{3,1}$, M. Bartelmann ${ }^{1}$, and N. Yoshida ${ }^{4,1}$ \\ 1 Max-Planck-Institut für Astrophysik, PO Box 1317, 85741 Garching, Germany \\ 2 Institut d'Astrophysique de Paris, 98 bis Bld Arago, 75014, Paris, France \\ 3 National Astronomical Observatory of Japan, Mitaka, Tokyo 181-8588, Japan \\ ${ }^{4}$ Harvard-Smithsonian Center for Astrophysics, 60 Garden Street, Cambridge MA 02138, USA
}

Received 4 October 2002 / Accepted 17 March 2003

\begin{abstract}
The systematic magnification of background sources by the weak gravitational-lensing effects of foreground matter, also called cosmic magnification, is becoming an efficient tool both for measuring cosmological parameters and for exploring the distribution of galaxies relative to the dark matter. We extend here the formalism of magnification statistics by estimating the contribution of second-order terms in the Taylor expansion of the magnification and show that the effect of these terms was previously underestimated. We test our analytical predictions against numerical simulations and demonstrate that including second-order terms allows the accuracy of magnification-related statistics to be substantially improved. We also show, however, that both numerical and analytical estimates can provide only lower bounds to real correlation functions, even in the weak lensing regime. We propose to use count-in-cells estimators rather than correlation functions for measuring cosmic magnification since they can more easily be related to correlations measured in numerical simulations.
\end{abstract}

Key words. cosmology: gravitational lensing - cosmology: large-scale structure of Universe

\section{Introduction}

Gravitational lensing by large-scale structures magnifies sources and distorts their images. The systematic distortion of faint background galaxies near matter overdensities, the cosmic shear, has been measured by several groups in the past few years (Bacon et al. 2000, 2002; Hämmerle et al. 2002; Hoekstra et al. 2002; Kaiser et al. 2000; Maoli et al. 2001; Réfrégier et al. 2002; Rhodes et al. 2001; Van Waerbeke et al. 2000, 2001, 2002; Wittman et al. 2000). It was found to be in remarkable agreement with theoretical predictions based on the Cold Dark Matter model, and has already provided new constraints on cosmological parameters (Van Waerbeke et al. 2001).

In a similar way, systematic magnifications of background sources near foreground matter overdensities, the cosmic magnification, can be measured and can provide largely independent constraints on cosmological parameters (Ménard \& Bartelmann 2002; Ménard et al. 2002). Gravitational magnification has two effects: first, the flux received from distant sources is increased, and the solid angle in which they appear is stretched, thus their density is diluted. The net result of these competing effects depends on how the loss of sources due to dilution is balanced by the gain of sources due to flux magnification. Sources with flat luminosity functions, like faint galaxies, are depleted by cosmic magnification, while the number

Send offprint requests to: $\mathrm{B}$. Ménard,

e-mail: menard@mpa-garching.mpg.de density of sources with steep luminosity functions, like quasars, is increased. Thus, cosmic magnification gives rise to apparent angular cross-correlations between background sources and foreground matter overdensities which are physically completely uncorrelated with the sources. These overdensities can be traced by using the distribution of foreground galaxies.

Numerous studies have demonstrated the existence of quasar-galaxy correlations on angular scales ranging from one arcminute to about one degree, as expected from cosmic lensing (for a review, see Bartelmann \& Schneider 2001; also Guimarães et al. 2001). In many cases, the measured correlation amplitudes have been higher than the theoretical predictions, however a number of non-detections have also been reported, leaving the true amplitude of the effect unclear from the observational point of view.

While cosmic shear can directly be related to observable quantities like image ellipticities, the theoretical interpretation of cosmic magnification involves several approximations:

- the luminosity function of the sources is described by a power-law over the range probed by the flux limit of the observation; and

- the magnification is assumed to fall into the weak lensing regime, i.e. to deviate weakly from unity. Thus, the magnification can with sufficient accuracy be approximated by its first-order Taylor expansion and its deviation from unity becomes proportional to the lensing convergence alone. 
While the first assumption is comfortably satisfied, in particular for quasars, the validity of the second needs to be verified. This is the goal of the present paper.

Our paper is structured as follows: first, we introduce the formalism of the effective magnification and its Taylor expansion in Sect. 2. We then describe a number of statistics related to the lensing convergence, and evaluate the amplitude of the second-order terms which appear in the Taylor expansion. In Sect. 3, we describe the numerical simulations we use to test our analytical results and estimate the accuracy of several approximations for the magnification. As an application, we investigate second-order effects on quasar-galaxy correlations in Sect. 4, and we summarise our results in Sect. 5.

\section{Formalism}

\subsection{Expanding the magnification}

Cosmic magnification can be measured statistically through characteristic changes in the number density of the background sources. Along a given line-of-sight, this effect depends on two quantities:

- the magnification factor $\mu$, which describes whether sources are magnified or demagnified, depending on whether the matter along their lines-of-sight is preferentially over- or underdense compared to the mean,

- and the logarithmic slope $\alpha$ of the source counts as a function of flux, which quantifies the amplitude of source number-count modifications due to flux magnification. As mentioned in the introduction, magnification by gravitational lensing not only increases the observed flux, but also stretches the sky, thus the number density of sources on a magnified patch of the sky is reduced. The net magnification effect, called magnification bias, depends on the balance between the number of sources lost by dilution and gained by flux magnification. The steeper the number-count function of the sources is, the more pronounced is the magnification bias.

If the number-count function of the background sources can be described as a power law in a sufficiently wide range around the flux limit of the observation, the magnification bias is quantified by the effective magnification $\mu^{\alpha-1}$. It directly expresses the changes of the background source density caused by lensing through the relation

$n(>S, \boldsymbol{\theta})=\mu^{\alpha-1}(\boldsymbol{\theta}) n_{0}(>S)$

where $n_{0}(>S)$ is the intrinsic number-count function of sources whose observed flux exceeds $S$ in the absence of lensing, and $n(>S)$ is the corresponding number-count function in presence of lensing.

The local properties of the gravitational lens mapping are characterised by the convergence $\kappa$, which is proportional to the surface mass density projected along the line-of-sight, and the shear $\gamma$, which is a two-component quantity and describes the gravitational tidal field of the lensing mass distribution. The effective magnification is related to $\kappa$ and $\gamma$ through

$\mu^{\alpha-1}=\left[(1-\kappa)^{2}-|\gamma|^{2}\right]^{1-\alpha}$, where $|\gamma|=\left(\gamma_{1}^{2}+\gamma_{2}^{2}\right)^{1 / 2}$ is taken as the absolute value of the shear. In the weak-lensing regime, both $\kappa$ and $|\gamma|$ are small compared to unity, and the previous expression can be expanded in a Taylor series:

$\mu^{\alpha-1}=1+(\alpha-1)\left[2 \kappa+(2 \alpha-1) \kappa^{2}+|\gamma|^{2}\right]+O\left(\kappa^{3},|\gamma|^{3}\right)$.

Previous studies using analytical formulae for magnification statistics focused only on the first-order term of this expansion, i.e. they used the approximation $\mu^{\alpha-1} \approx 1+2(\alpha-1) \kappa$, which potentially causes the amplitude of the effect to be underestimated. In this section, we investigate the second-order terms in the expansion and estimate their contribution.

In doing so, we first note that $\kappa^{2}(\boldsymbol{\theta})$ and $|\gamma|^{2}(\boldsymbol{\theta})$ share the same statistical properties (e.g. Blandford et al. 1991), because both $\kappa$ and $\gamma$ are linear combinations of second-order derivatives of the lensing potential. The identity of their statistics is most easily seen in Fourier space. Since we will only deal with ensemble averages of the magnification later on, $\kappa^{2}$ and $|\gamma|^{2}$ can be combined into a single variable, which we denote by $\kappa$ for simplicity. Thus, we can write for our purposes,

$\mu^{\alpha-1}=1+2(\alpha-1)\left[\kappa+\alpha \kappa^{2}\right]+O\left(\kappa^{3}\right)$.

Observable effects are due to departures from the mean value of the magnification. Therefore, the relevant quantity to correlate is $\delta \mu^{\alpha-1}=\mu^{\alpha-1}-\left\langle\mu^{\alpha-1}\right\rangle$. Then, up to second order in $\kappa^{2}$, the autocorrelation function of the effective magnification is

$$
\begin{aligned}
\left\langle\delta \mu^{\alpha-1}(\boldsymbol{\phi}) \delta \mu^{\alpha-1}(\boldsymbol{\phi}+\boldsymbol{\theta})\right\rangle= & 4(\alpha-1)^{2}[\langle\kappa(\boldsymbol{\phi}) \kappa(\boldsymbol{\phi}+\boldsymbol{\theta})\rangle \\
& \left.+2 \alpha\left\langle\kappa(\boldsymbol{\phi}) \kappa^{2}(\boldsymbol{\phi}+\boldsymbol{\theta})\right\rangle\right]
\end{aligned}
$$

and the corresponding power spectrum can be expanded in a similar way,

$P_{\mu^{\alpha-1}}(s)=4(\alpha-1)^{2}\left[P_{K}(s)+2 \alpha P_{\mu, 2}(s)\right] ;$

the power spectrum $P_{\mu, 2}(s)$ will be defined in Eq. (16) below. The last two equations show that the importance of the secondorder terms in the expansion (3) increases as the number-count function of the background sources steepens, i.e. as $\alpha$ increases. In the following, we will use $\alpha=2$ unless stated otherwise. This value applies, for instance, to the number counts of bright quasars with $m_{\mathrm{B}}<19.5$ (Pei 1995). For simplicity, we abbreviate $\langle\delta \mu \delta \mu\rangle$ by $\langle\mu \mu\rangle$.

\subsection{Second and third-order correlations}

We will now estimate several $\kappa$-related statistical quantities needed in the Taylor expansion of the magnification. For this purpose, we first introduce the $\kappa$ projector such that

$\kappa(\boldsymbol{\theta})=\int_{0}^{w_{\mathrm{H}}} \mathrm{d} w p_{\kappa}(w) \delta\left[\boldsymbol{\theta} f_{K}(w), w\right]$

can be written as a weighted line-of-sight projection of the density contrast $\delta$ from the observer to the Hubble distance $w_{\mathrm{H}}$. The projector is

$$
\begin{aligned}
p_{\kappa}(w)= & \frac{3}{2} \Omega_{0}\left(\frac{H_{0}}{c}\right)^{2} \\
& \times \int_{w}^{w_{\mathrm{H}}} \frac{\mathrm{d} w^{\prime}}{a(w)} n_{\mathrm{S}}\left(w^{\prime}\right) \frac{f_{K}(w) f_{K}\left(w^{\prime}-w\right)}{f_{K}\left(w^{\prime}\right)},
\end{aligned}
$$


where $w$ is the radial coordinate distance, $f_{K}(w)$ is the comoving angular-diameter distance, $n_{\mathrm{S}}(w)$ is the normalised distance distribution of the sources, and $a(w)$ is the cosmological scale factor. Using Limber's equation, we can then relate the autocorrelation function of $\kappa$ to the dark-matter power spectrum $P_{\delta}$,

$$
\begin{aligned}
\langle\kappa(\boldsymbol{\phi}) \kappa(\boldsymbol{\phi}+\boldsymbol{\theta})\rangle= & \int \mathrm{d} w \frac{p_{\kappa}^{2}(w)}{f_{K}^{2}(w)} \\
& \times \int \frac{s \mathrm{~d} s}{2 \pi} P_{\delta}\left(\frac{s}{f_{k}(w)}, w\right) J_{0}(s \theta),
\end{aligned}
$$

where $J_{0}$ is the zeroth-order Bessel function, and the power spectrum $P_{K}$ corresponding to this correlation function is

$P_{K}(s)=\int \mathrm{d} w \frac{p_{\kappa}^{2}(w)}{f_{K}^{2}(w)} P_{\delta}\left(\frac{s}{f_{k}(w)}, w\right)$.

As indicated by Eq. (5), the estimation of second-order terms requires the computation of the cross-correlation between $\kappa$ and $\kappa^{2}$. We do this by first introducing a three-point correlation function for $\kappa$ and then identifying two of its three points. As usual, we define the three-point function by

$z_{\kappa}\left(\boldsymbol{\theta}_{1}, \boldsymbol{\theta}_{2}\right)=\left\langle\kappa(\boldsymbol{\phi}) \kappa\left(\boldsymbol{\phi}+\boldsymbol{\theta}_{1}\right) \kappa\left(\boldsymbol{\phi}+\boldsymbol{\theta}_{2}\right)\right\rangle$.

Using the $\kappa$ projector defined in (8), we can then write

$$
\begin{aligned}
z_{\kappa}\left(\boldsymbol{\theta}_{1}, \boldsymbol{\theta}_{2}\right)= & \int \mathrm{d} w_{1} p_{\kappa}\left(w_{1}\right) \int \mathrm{d} w_{2} p_{\kappa}\left(w_{2}\right) \int \mathrm{d} w_{3} p_{\kappa}\left(w_{3}\right) \\
& \times\left\langle\delta\left[f_{K}\left(w_{1}\right) \boldsymbol{\phi}, w_{1}\right] \delta\left[f_{K}\left(w_{2}\right)\left(\boldsymbol{\phi}+\boldsymbol{\theta}_{1}\right), w_{2}\right]\right. \\
& \left.\times \delta\left[f_{K}\left(w_{3}\right)\left(\boldsymbol{\phi}+\boldsymbol{\theta}_{2}\right), w_{3}\right]\right\rangle .
\end{aligned}
$$

Next, we employ the approximation underlying Limber's equation, which asserts that the coherence length of the density fluctuation field is much smaller than the scales on which the projector $p_{\kappa}$ varies appreciably. Finally, we insert the expression for the bispectrum of the dark-matter fluctuations detailed in Appendix A, and find

$$
\begin{aligned}
z_{\kappa}\left(\boldsymbol{\theta}_{1}, \boldsymbol{\theta}_{2}\right)= & \int \mathrm{d} w p_{\kappa}^{3}(w) \int \frac{\mathrm{d}^{2} k_{1}}{(2 \pi)^{2}} \mathrm{e}^{\mathrm{i} \boldsymbol{k}_{1} \cdot \boldsymbol{\theta}_{1} f_{K}(w)} \int \frac{\mathrm{d}^{2} k_{2}}{(2 \pi)^{2}} \\
& \times B_{\delta}\left(\boldsymbol{k}_{1}, \boldsymbol{k}_{2},-\boldsymbol{k}_{1}-\boldsymbol{k}_{2}, w\right) \mathrm{e}^{\mathrm{i} \boldsymbol{k}_{2} \cdot \boldsymbol{\theta}_{2} f_{K}(w)},
\end{aligned}
$$

where $B_{\delta}\left(\boldsymbol{k}_{1}, \boldsymbol{k}_{2}, \boldsymbol{k}_{3}\right)$ is defined by

$$
\left\langle\hat{\delta}\left(\boldsymbol{k}_{1}\right) \hat{\delta}\left(\boldsymbol{k}_{2}\right) \hat{\delta}\left(\boldsymbol{k}_{3}\right)\right\rangle=\delta_{D}\left(\boldsymbol{k}_{1}+\boldsymbol{k}_{2}+\boldsymbol{k}_{3}\right) B_{\delta}\left(\boldsymbol{k}_{1}, \boldsymbol{k}_{2}, \boldsymbol{k}_{3}\right) .
$$

Then, using Eq. (12) and identifying two points of the threepoint correlation function $\boldsymbol{\theta}_{1} \rightarrow \boldsymbol{\theta}_{2} \equiv \boldsymbol{\theta}$ (or equivalently $\boldsymbol{\theta}_{1}$ or $\left.\theta_{2} \rightarrow \mathbf{0}\right)$, we find

$$
\begin{aligned}
\left\langle\kappa(\boldsymbol{\phi}) \kappa^{2}(\boldsymbol{\phi}+\boldsymbol{\theta})\right\rangle= & \int \mathrm{d} w p_{\kappa}^{3}(w) \int \frac{\mathrm{d}^{2} k_{1}}{(2 \pi)^{2}} \mathrm{e}^{\mathrm{i} \boldsymbol{k}_{1} \cdot \boldsymbol{\theta} f_{K}(w)} \\
& \times \int \frac{\mathrm{d}^{2} k_{2}}{(2 \pi)^{2}} B_{\delta}\left(\boldsymbol{k}_{1}, \boldsymbol{k}_{2},-\boldsymbol{k}_{1}-\boldsymbol{k}_{2}, w\right) .
\end{aligned}
$$

The term $\left\langle\kappa(\phi) \kappa^{2}(\boldsymbol{\phi}+\boldsymbol{\theta})\right\rangle$ is a function of $\theta$ only. Its contribution $P_{\mu, 2}(s)$ to the power spectrum of the magnification is given by the inverse Fourier transform of Eq. (15), which reads

$$
\begin{aligned}
P_{\mu, 2}(s)= & \int \mathrm{d} w \frac{p_{K}^{3}(w)}{f_{K}^{4}(w)} \int \frac{\mathrm{d}^{2} s^{\prime}}{(2 \pi)^{2}} \\
& \times B_{\delta}\left(\frac{\boldsymbol{s}^{\prime}}{f_{k}(w)}, \frac{\boldsymbol{s}}{f_{k}(w)}, \frac{-\boldsymbol{s}^{\prime}-\boldsymbol{s}}{f_{k}(w)}, w\right) .
\end{aligned}
$$

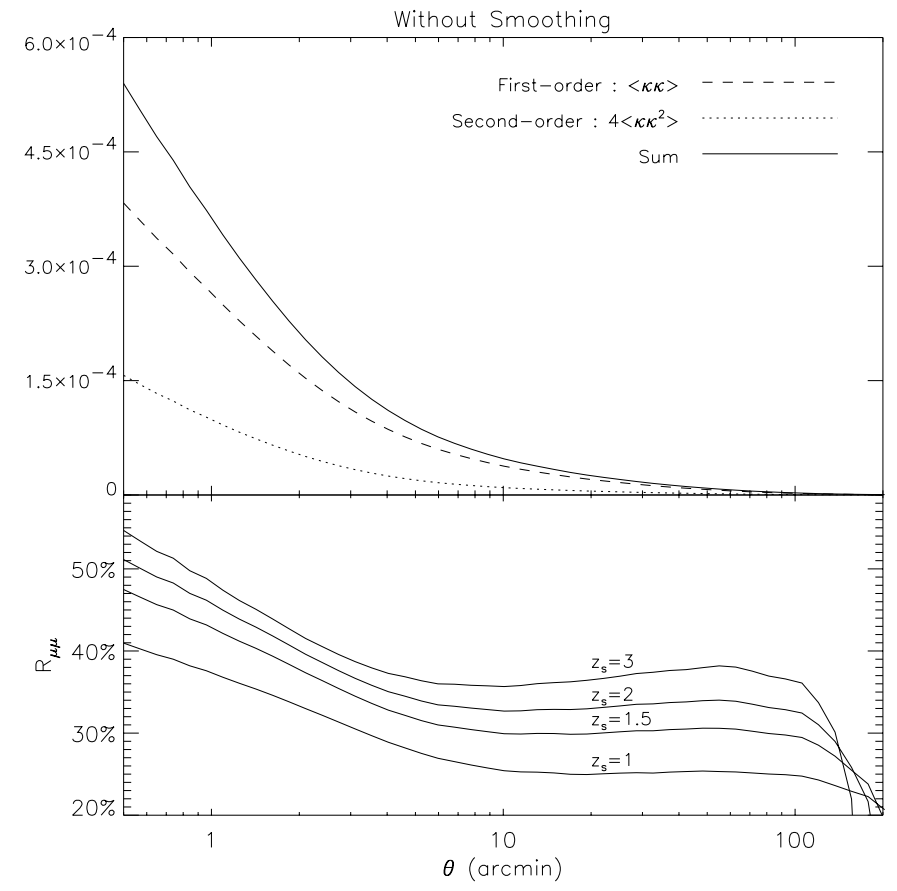

Fig. 1. The upper panel shows the amplitude of the two first terms of the Taylor expansion of the magnification autocorrelation, namely $\langle\kappa(\phi) \kappa(\boldsymbol{\phi}+\boldsymbol{\theta})\rangle$ (dashed line) and $4\left\langle\kappa(\boldsymbol{\phi}) \kappa^{2}(\boldsymbol{\phi}+\boldsymbol{\theta})\right\rangle$ (dotted line), using a source redshift of unity. The sum of these two terms is shown as the solid line. The lower panel details the relative contribution $R_{\mu \mu}$ of the second-order term for different source redshifts. The figure shows that the lowest-order approximation $\mu \approx 1+2 \kappa$ misses a substantial part of the amplitude of the magnification autocorrelation function. Given the accuracy of the bispectrum fitting formula, $R_{\mu \mu}$ is accurate to $\sim 2 \%$.

\subsection{Results and predictions}

We can now numerically evaluate the first two contributions to the Taylor expansion of the magnification autocorrelation function defined in Eq. (5). As mentioned before, we use $\alpha=2$ here.

For evaluating the correlation functions, we use a CDM power spectrum in a spatially flat Universe parameterised with $\Omega_{0}=0.3, \sigma_{8}=0.9, h=0.7$ and $\Gamma=0.21$. The non-linear evolution of the power spectrum and the bispectrum are computed according to the formalisms developed by Peacock \& Dodds (1996) and Scoccimarro et al. (2000), see Appendix A. The upper panel of Fig. 1 shows the first- and second-order contributions (dashed and dotted lines, respectively) to the Taylor expansion of the magnification for a fixed source redshift of $z_{\mathrm{s}}=$ 1. The sum of the two contributions is shown by the solid line. The figure shows that the contribution of the second-order term reaches an amplitude of more than $30 \%$ of the first-order term on angular scales smaller than one arcminute. According to Eq. (5) which describes the Taylor expansion of the magnification autocorrelation, we define the contribution of the secondorder relative to the first-order term as

$R_{\mu \mu}(\theta)=\frac{2 \alpha\left\langle\kappa(\phi) \kappa^{2}(\boldsymbol{\phi}+\boldsymbol{\theta})\right\rangle}{\langle\kappa(\boldsymbol{\phi}) \kappa(\boldsymbol{\phi}+\boldsymbol{\theta})\rangle}$.

The lower panel shows this ratio in per cent for different source redshifts as a function of angular scale. From the lower to the 
upper curves, the source redshifts are 1, 1.5, 2 and 3. For each source redshift, the contribution of the second term exhibits a similar dependence on angular scale:

- on scales larger than a few degrees, the contribution drops to negligible values;

- effects become relevant on smaller scales, with a fairly constant amplitude from a few degrees down to around 10 arcmin;

- on yet smaller scales, the second-order contribution increases steeply, due to the non-linear evolution of the density field. For sources at redshift 2, the amplitude of the second term reaches half of the amplitude of the first term below one arcmin.

Thus, given the amplitude of $R_{\mu \mu}$, the correcting term introduced in Eq. (5) is relevant and must be taken into account for describing the magnification autocorrelation with an accuracy better than $30 \%-50 \%$ on scales smaller than a few degrees.

So far, we have only investigated the amplitude contributed by the second-order term. In order to estimate the remaining contributions of all missing terms of the magnification expansion, we will now use numerical simulations allowing a direct computation of $\mu$ as a function of the convergence $\kappa$ and the shear $\gamma$.

\section{Magnification statistics from numerical simulations}

On sub-degree scales, lensing effects due to non-linearities in the density field can only be approximated using analytical fitting formulae (Peacock \& Dodds 1996; Scoccimarro \& Couchman 2001) as seen above. A full description requires numerical simulations (White \& Hu 2000).

\subsection{The ray-tracing simulation}

For testing the theoretical predictions we performed ray-tracing experiments in a Very Large $N$-body Simulation (VLS) recently carried out by the Virgo Consortium (Jenkins et al. 2001; and see also Yoshida et al. 2001 for simulation details) ${ }^{1}$.

The simulation was performed using a parallel $\mathrm{P}^{3} \mathrm{M}$ code (MacFarland et al. 1998) with a force softening length of $l_{\text {soft }} \sim$ $30 h^{-1} \mathrm{kpc}$. The simulation employed $512^{3} \mathrm{CDM}$ particles in a cubic box of $479 h^{-1} \mathrm{Mpc}$ on a side. It uses a flat cosmological model with a matter density $\Omega_{0}=0.3$, a cosmological constant $\Omega_{\Lambda}=0.7$, and a Hubble constant $h=0.7$. The initial matter power spectrum was computed using CMBFAST (Seljak \& Zaldarriaga 1996) assuming a baryonic matter density of $\Omega_{\mathrm{b}}=0.04$. The particle mass $\left(m_{\text {part }}=6.86 \times 10^{10} h^{-1} M_{\odot}\right)$ of the simulation is sufficiently small to guarantee practically no discreteness effects on dark-matter clustering on scales down to the softening length in the redshift range of interest for our purposes (Hamana et al. 2002).

The multiple-lens plane ray-tracing algorithm we used is detailed in Hamana \& Mellier (2001; see also

\footnotetext{
1 The ray-tracing data are available from T. Hamana on request, hamanatk@cc.nao.ac.jp
}

Bartelmann \& Schneider 1992; Jain et al. 2000 for the theoretical basics); we thus describe only aspects specific to the VLS $N$-body data in the following. In order to generate the density field between $z=0$ and $z \sim 3$, we use a stack of ten snapshot outputs from two runs of the $N$-body simulation, which differ only in the realisation of the initial fluctuation field. Each cubic box is divided into 4 sub-boxes of $479^{2} \times 119.75 \mathrm{~h}^{-3} \mathrm{Mpc}^{3}$ with the shorter box side being aligned with the line-of-sight direction. The $N$-body particles in each sub-box are projected onto the plane perpendicular to the shorter box side and thus to the line-of-sight direction. In this way, the particle distribution between the observer and $z \sim 3$ is projected onto 38 lens planes separated by $119.75 h^{-1} \mathrm{Mpc}$. Note that in order to minimise the difference in redshift between a lens plane and an output of $N$-body data, only one half of the outputs (i.e. two sub-boxes) at $z=0$ are used.

The particle distribution on each plane is converted into the surface density field on either a $1024^{2}$ or $2048^{2}$ regular grid using the triangular shaped cloud (TSC) assignment scheme (Hockney \& Eastwood 1988). The two grid sizes are adopted for the following reasons:

- the $1024^{2}$ grid is chosen to maintain the resolution provided by the $N$-body simulation and removing at the same time the shot noise due to discreteness in the $N$-body simulation. Its computation follows the procedure described in Hamana $\&$ Mellier (2001) and Jain et al. (2000). The corresponding outputs will be labelled with large-scale smoothing in the following.

- the $2048^{2}$ grid is also chosen to examine effects of smallscale nonlinear structures which are smoothed in the largescale smoothing simulation. We should, however, note that in this case the shot noise is not sufficiently removed. Actually, the shot-noise power spectrum amplitude exceeds the convergence power spectrum on scales below $\sim 1$ arcmin. In the following, therefore, we will only consider measured correlation functions on scales larger than 1 arcmin. The corresponding outputs will be labelled with small-scale smoothing below.

Having produced surface density fields on all lens planes, $1024^{2}$ rays are traced backwards from the observer's point using the multiple-lens plane algorithm (e.g. Schneider et al. 1992). The initial ray directions are set on $1024^{2}$ grids with a grid size of 0.25 arcmin, thus the total area covered by rays is $4.27^{2}$ square degrees. We produced 36 realizations of the underlying density field by randomly shifting the simulation boxes in the direction perpendicular to the line-of-sight using the periodic boundary conditions of the $N$-body boxes. Note that the lens planes coming from the same box are shifted in the same way in order to maintain the clustering of matter in the box.

We point out that second and higher-order statistics of point-source magnifications are generally ill-defined in presence of caustic curves because the differential magnification probability distribution asymptotically decreases as $\mu^{-2}$ for large $\mu$ (see Fig. 2). This is a generic feature of magnification near caustics and is thus independent of the lens model. Strong lensing effects on point sources near caustic curves 


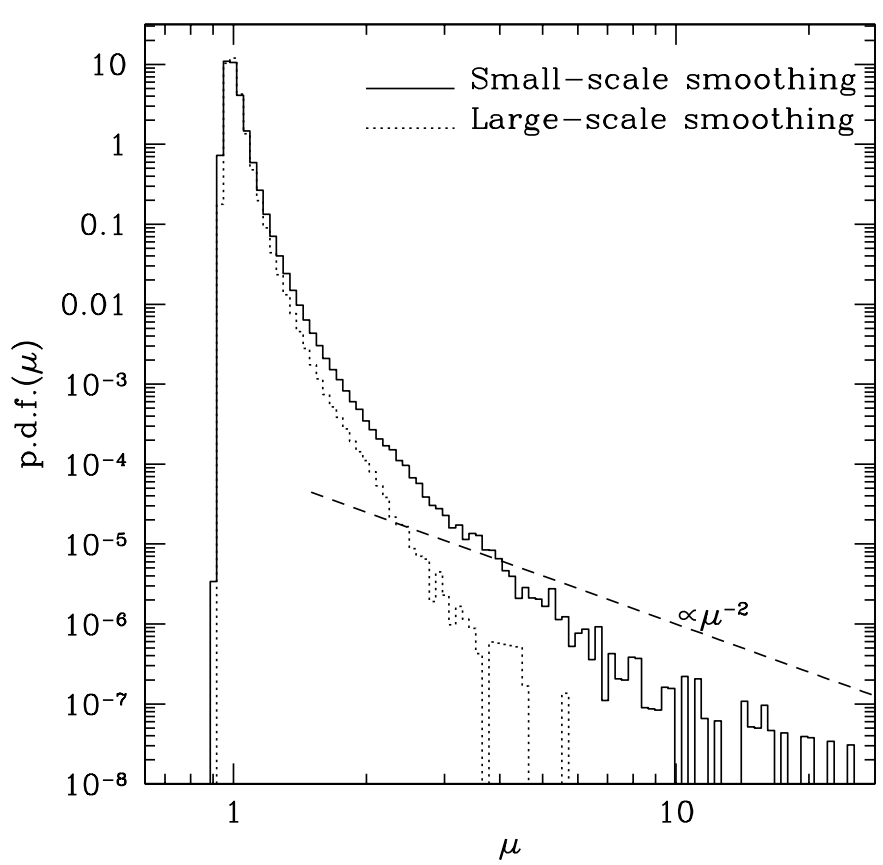

Fig. 2. Probability distribution of the magnification for our small- and large-scale smoothing simulations, assuming sources at redshift unity. The power law tail behaviour $\left(\propto \mu^{-2}\right)$ found in the small-scale smoothing indicates the existence of caustics, while for large-scale smoothing, no prominent tail is shown which suggests caustics do not play a noticeable role.

give rise to rare, but arbitrarily high magnification values in the simulations, and therefore the variance of the measured statistics of $\mu$ cannot be defined. However, the smoothing procedure introduced above allows this problem to be removed because it smoothes out high density regions in the dark matter distribution and thus the fractional area of high magnification decreases. In reality, infinite magnifications do not occur, for two reasons. First, each astrophysical source is extended and its magnification (given the surface brightness-weighted point-source magnification across its solid angle) remains finite. Second, even point sources would be magnified by a finite value since for them, the geometrical-optics approximation fails near critical curves and a wave-optics description leads to a finite magnification (Schneider et al. 1992, Chap. 7).

\subsection{Filtering}

The computation of correlation functions from numerical simulations is mainly affected by two effects; on large scales by the finite box size of the dark matter simulation, and on small scales by the grid size used for computing the surface density field from the particle distribution. These boundaries set the limits for the validity of correlation functions measured in numerical simulations. In other words, this means that measuring a correlation function on a given scale is relevant only if this scale falls within the range of scales defined by the simulation. As shown in the previous section, our method for computing the cross-correlation between $\kappa$ and $\kappa^{2}$ consists of first computing a three-point correlation function $\left\langle\kappa(\phi) \kappa\left(\phi+\boldsymbol{\theta}_{1}\right) \kappa\left(\boldsymbol{\phi}+\boldsymbol{\theta}_{2}\right)\right\rangle$, and then identifying two of its three points. In such a case, one of the correlation lengths of the triple correlator becomes zero, thus necessarily smaller than the smallest relevant scales in any simulation. This prevents us from using any numerical simulation for directly comparing the results.

In order to avoid this problem, and for comparing our analytical with numerical results, we will introduce an effective smoothing into the theoretical calculations, such that each value of $\kappa$ at a given position $\boldsymbol{\theta}$ is evaluated by averaging the $\boldsymbol{\kappa}$-values in a disk of radius $\boldsymbol{\theta}_{\mathrm{S}}$ centred on $\boldsymbol{\theta}$. Indeed, the limit imposed by the grid size of the simulation gives rise to an unavoidable smoothing-like effect which cancels all information coming from scales smaller than a corresponding smoothing scale $\boldsymbol{\theta}_{\mathrm{S}}$. For this purpose, we introduce a smoothed three-point correlator,

$$
\begin{aligned}
& z_{\text {smooth }}\left(\boldsymbol{\theta}_{2}-\boldsymbol{\theta}_{1}, \boldsymbol{\theta}_{3}-\boldsymbol{\theta}_{1}\right)=\left\langle\kappa\left(\boldsymbol{\theta}_{1}\right) \kappa\left(\boldsymbol{\theta}_{2}\right) \kappa\left(\boldsymbol{\theta}_{3}\right)\right\rangle_{\theta_{\mathrm{S}}} \\
& =\int \mathrm{d} \boldsymbol{\theta}_{1}^{\prime} \int \mathrm{d} \boldsymbol{\theta}_{2}^{\prime} \int \mathrm{d} \boldsymbol{\theta}_{3}^{\prime}\left\langle\kappa\left(\boldsymbol{\theta}_{1}^{\prime}\right) \kappa\left(\boldsymbol{\theta}_{2}^{\prime}\right) \kappa\left(\boldsymbol{\theta}_{3}^{\prime}\right)\right\rangle \\
& \times W_{\theta_{\mathrm{S}}}\left(\boldsymbol{\theta}_{1}^{\prime}-\boldsymbol{\theta}_{1}\right) W_{\theta_{\mathrm{S}}}\left(\boldsymbol{\theta}_{2}^{\prime}-\boldsymbol{\theta}_{2}\right) W_{\theta_{\mathrm{S}}}\left(\boldsymbol{\theta}_{3}^{\prime}-\boldsymbol{\theta}_{3}\right),
\end{aligned}
$$

where the function $W_{\theta_{\mathrm{S}}}\left(\theta^{\prime}\right)$ is a normalised top-hat window of radius $\theta_{\mathrm{S}}$. Introducing this smoothing scheme into the expression for $\left\langle\kappa(\phi) \kappa^{2}(\boldsymbol{\phi}+\boldsymbol{\theta})\right\rangle$ yields

$$
\begin{aligned}
z_{\text {smooth }}\left(\theta_{\mathrm{S}}\right)= & \int \mathrm{d} w \frac{p_{K}^{3}(w)}{f_{K}^{4}(w)} \int \frac{\mathrm{d}^{2} s_{1}}{(2 \pi)^{2}} \int \frac{\mathrm{d}^{2} s_{2}}{(2 \pi)^{2}} \\
& \times I\left(s_{1} \theta_{\mathrm{S}}\right) I\left(s_{2} \theta_{\mathrm{S}}\right) I\left(\left|s_{1}+s_{2}\right| \theta_{\mathrm{S}}\right) \\
& \times B_{\delta}\left(\frac{s_{1}}{f_{k}(w)}, \frac{s_{2}}{f_{k}(w)}, \frac{-s_{1}-s_{2}}{f_{k}(w)}, w\right) \mathrm{e}^{\mathrm{i} s_{1} \theta}
\end{aligned}
$$

where $\mathrm{I}(x)=2 \frac{\mathrm{J}_{1}(x)}{x}$. Similarly, introducing the smoothing scheme into the two-point correlation function gives

$$
\begin{aligned}
w_{\text {smooth }}\left(\left|\boldsymbol{\theta}_{2}-\boldsymbol{\theta}_{1}\right|\right)= & \left\langle\kappa\left(\boldsymbol{\theta}_{1}\right) \kappa\left(\boldsymbol{\theta}_{2}\right)\right\rangle_{\theta_{\mathrm{S}}} \\
= & \int \mathrm{d} w \frac{p_{\kappa}^{2}(w)}{f_{K}^{2}(w)} \int \frac{\mathrm{d}^{2} s}{(2 \pi)^{2}} P\left(\frac{s}{f_{K}(w)}, w\right) \\
& \times\left|I\left(s \theta_{\mathrm{S}}\right)\right|^{2} \mathrm{e}^{\mathrm{i} \boldsymbol{\theta} \boldsymbol{\theta}}
\end{aligned}
$$

The effective smoothing scale depends on two parameters:

- the evolution of the apparent grid size of the simulation as a function of redshift, and

- the radial selection function of the dark-matter field whose correlation function has to be measured.

These quantities are plotted in Fig. 3. In order to use a unique smoothing scale valid on the final convergence map, we define the effective angular smoothing scale by

$\theta_{\mathrm{S}}=\int \mathrm{d} z W(z) \theta_{\text {grid }}(z)$

where $W(z)$ is the relevant normalised selection function along the line-of-sight. Measuring $w_{\mathrm{s}}$ means probing the power spectrum along the line-of-sight, weighted by $p_{\kappa}^{2}(z)$. Therefore, we will use $W(z)=D_{+}^{2}(z) p_{\kappa}^{2}(z)$, where $D_{+}(z)$ is the growth factor. In a similar way, we will use $W(z)=D_{+}^{4}(z) p_{\kappa}^{3}(z)$ for measuring $z_{\text {smooth }}$. The numerical values of the corresponding effective angles are presented in Table 1. 


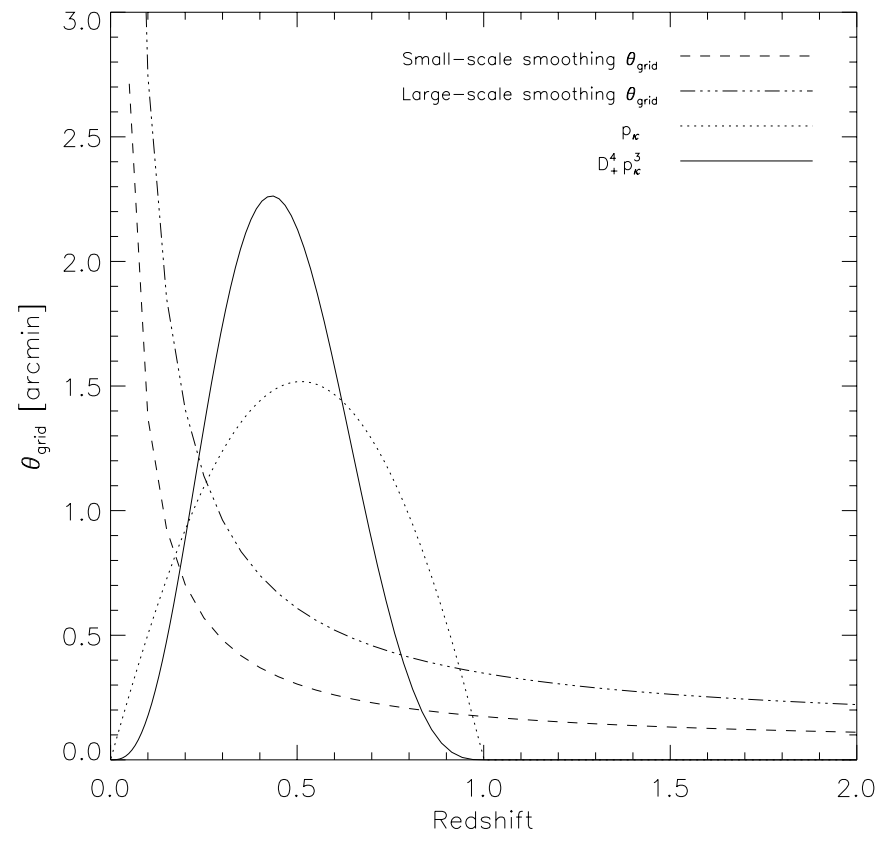

Fig. 3. Smoothing angle of the simulation as a function of redshift for the two ray-tracing schemes. In order to show the relevant quantities leading to the effective smoothing angle, we overplot the weighting function $W(z)=D_{+}^{4}(z) p_{\kappa}^{3}(z)$ (see Eqs. (8) and (21)).

Table 1. Effective smoothing angles in arcminutes for $w_{s}$ and $z_{s}$ computed from Eq. (21) as a function of simulation resolution.

\begin{tabular}{ccc}
\hline \hline & small-scale smoothing & large-scale smoothing \\
\hline$w_{\text {smooth }}$ & $\theta_{\mathrm{S}}=0.40$ & $\theta_{\mathrm{S}}=0.80$ \\
$z_{\text {smooth }}$ & $\theta_{\mathrm{S}}=0.39$ & $\theta_{\mathrm{S}}=0.78$ \\
\hline
\end{tabular}

The second important difference between analytical calculations and measurements in numerical simulations is the finite box size effect. Indeed, the analytical correlation functions presented above were computed taking into account all modes in the power spectrum. However, the finite size of the box used in the simulation introduces an artificial cutoff in the power spectrum since wavelengths larger than the box size are not sampled by the simulation. This effect can also be taken into account in the analytical calculations by simply cancelling all the power on wavelengths with wave number $k<k_{\min }$. The boxes we use have a comoving size of $480 \mathrm{~h}^{-1} \mathrm{Mpc}$ which corresponds to $k_{\min }=0.013 \mathrm{~h} \mathrm{Mpc}^{-1}$.

\subsection{Comparing $\langle\kappa \kappa\rangle$ and $\left\langle\kappa \kappa^{2}\right\rangle$}

With the help of the filtering schemes introduced in the previous section, we can now compare our theoretical predictions with correlation functions measured from the numerical simulations. We first compare the amplitude and angular variation of the two first terms of the Taylor expansion of the magnification separately. In the next section, we will then compare their sum to the total magnification fully computed from the simulation.

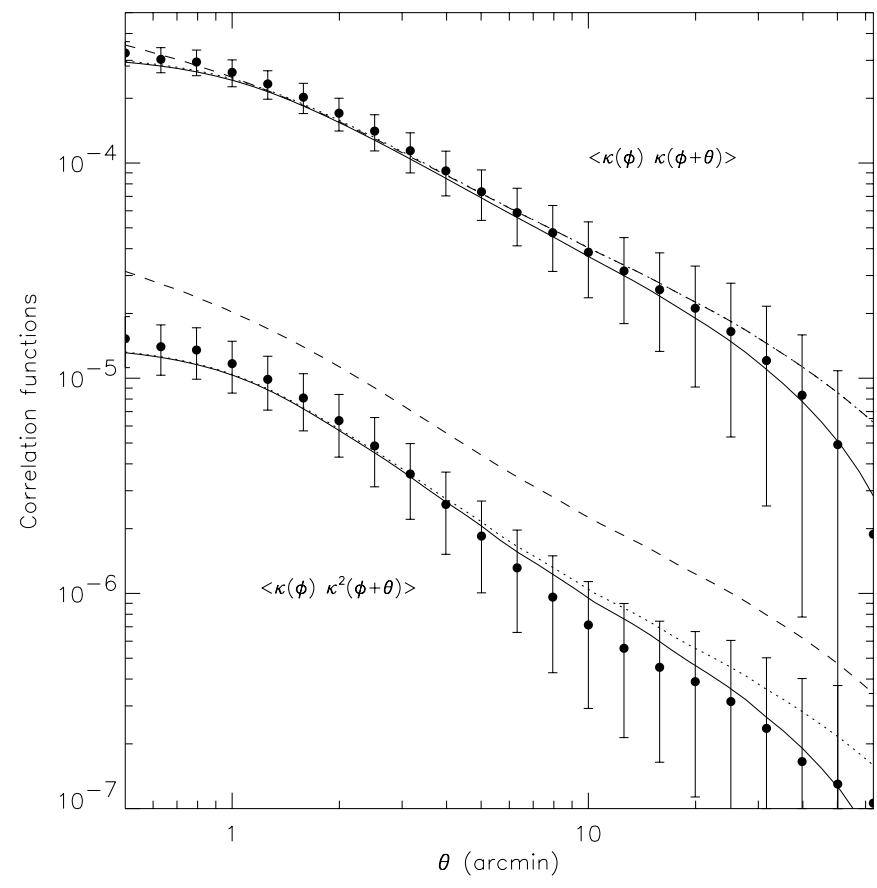

Fig. 4. Comparison between theoretical predictions and measurements from numerical simulations assuming sources at redshift unity. The upper and lower curves show $\langle\kappa \kappa\rangle$ and $\left\langle\kappa \kappa^{2}\right\rangle$, respectively. The points are measurements from the large-scale smoothing simulations, with the error bars showing the variance among 36 different realisations. The dotted lines show the analytical computations taking into account the smoothing scale of the simulation. The solid lines additionally include a cut in the power spectrum for cancelling the wavelengths not covered by the simulation. The dashed line presents the same statistics without any smoothing. Obviously, the smoothing effects are crucial for the $\left\langle\kappa \kappa^{2}\right\rangle$ cross-correlation.

In Fig. 4, we overplot analytical and numerical results. The upper curve shows the autocorrelation function of $\kappa$ as a function of angular scale. We plot in circles the average measurement from 36 realisations of the simulation, and the corresponding 1- $\sigma$ error bars to show the accuracy of the numerical results as a function of angular scale. The solid line shows the analytical prediction, including effective smoothing and an artificial cut of the power at scales below $k_{\min }$. The agreement is good on all scales. For comparison, the dotted line shows the result if we do not impose the large-wavelength cut, and the dashed line is the result if no cut and no smoothing are applied. In both cases, the deviations from the fully filtered calculation remain small since we are probing angular scales within the range allowed by the simulation.

The lower curves in Fig. 4 show a quantity proportional to the second-order correction of the Taylor expansion, namely the correlation function $\left\langle\kappa \kappa^{2}\right\rangle$. In the same way as before, the circles show average measurements from 36 realisations, and the error bars denote the corresponding $1-\sigma$ deviation. The prediction including smoothing and small-wavelength cut (solid line) shows a relatively good agreement given the expected accuracy of the bispectrum fitting formula, which is approximately $15 \%$ (Scoccimarro \& Couchman 2000). This time, including smoothing changes the amplitude dramatically, and 
this effect affects all scales (see the dashed line). As discussed before, this is expected since we are measuring a three-point correlator on triangles which have one side length smaller than the angular grid size of the simulation. Finally, as shown by the difference between the dotted and solid lines, cancelling the power on scales where $k<k_{\min }$ again improves the agreement on large scales.

The agreement between our analytical and numerical computations of $\langle\kappa \kappa\rangle$ and $\left\langle\kappa \kappa^{2}\right\rangle$ demonstrates the validity of the formalism introduced in Sect. 2 as well as the choice of the effective smoothing scale (Eq. (21)) for describing the secondorder term in the Taylor expansion of the magnification.

\subsection{Deviations from simulated $\mu$-statistics}

We now want to investigate how well the second-order expansion describes the full magnification expression (2) which can be computed using maps of $\kappa, \gamma$ and $\omega$ (a net rotation term which arises from lens-lens coupling and the lensing deflection of the light ray path; see Van Waerbeke et al. 2001b) obtained from the simulations (see Hamana et al. 2000 for more detail).

Before doing so, we recall that the amplitude of the magnification autocorrelation measured from the simulation depends on the smoothing scale, as seen in Sect. 3.2, since $\mu$ is nonlinear in the density field. Therefore, all the following comparisons are valid for a given effective smoothing length only.

We further emphasise that two problems will complicate this comparison. First, our analytical treatment is valid in the weak-lensing regime only, i.e. as long as convergence and shear are small compared to unity, $\kappa \ll 1,|\gamma| \ll 1$. While most light rays traced through the numerical simulations are indeed weakly lensed, a non-negligible fraction of them will experience magnifications well above two, say. Such events are restricted to small areas with high overdensities and thus affect the magnification statistics only at small angular scales. Second, a separate problem sets in if and where caustics are formed. The magnification of light rays going through caustics is infinite, and the magnification probability distribution near caustics drops like $\mu^{-2}$ for $\mu \rightarrow \infty$. As noted above, second- or higher-order statistics of $\mu$ then become meaningless because they diverge.

Departures of the numerical from the analytical results will thus have two distinct reasons, viz. the occurrence of non-weak magnifications which causes the analytical to underestimate the numerical results on small angular scales; and the formation of caustics, which causes second-order magnification statistics to break down entirely. Both effects will be demonstrated below. They can be controlled or suppressed in numerical simulations by smoothing, which makes lensing weaker, or by masking highly magnified light rays or regions containing caustics.

In Fig. 5, we plot with circles the autocorrelation function $\langle\mu(\boldsymbol{\phi}) \mu(\boldsymbol{\phi}+\boldsymbol{\theta})\rangle$ measured from the large- and small-scale smoothing simulations in the left and right panels, respectively. The presence of caustics is more pronounced in the case of small-scale smoothing than in the large-scale smoothing simulations. The dotted line shows the theoretical prediction given by the first-order term of the Taylor expansion, namely $4\langle\kappa(\phi) \kappa(\phi+\theta)\rangle$. This yields a low estimate of the correlation, with a discrepancy of order $10 \%$ on large scales, and more than $20 \%$ below a few arcminutes.

As expected from the preceding discussion, this level of discrepancy also depends on the effective smoothing scale and can increase if simulations with a smaller grid size are used. Estimating the contribution of the two lowest-order terms of $\mu^{\alpha-1}$, we computed in Sect. 2.3 a lower bound to this discrepancy for a real case without smoothing, and found it to reach a level of $25 \%$ at large scales, and above $30 \%$ below a few arcminutes. The smoothed results taking the additional contribution of the second-order term into account are plotted as solid lines, and give a much better agreement, as expected. To quantify this in more detail, the lower panels of the figure show several contributions compared to the first-order term, i.e. to $4\langle\kappa \kappa\rangle$.

- The symbols show the additional amplitude of the magnification statistics measured from the simulation, compared to the first-order term also obtained from the simulation,

$R_{\mu \mu}^{\text {num }}=\frac{\langle\mu(\boldsymbol{\phi}) \mu(\boldsymbol{\phi}+\boldsymbol{\theta})\rangle_{\mathrm{num}}}{4\langle\kappa(\boldsymbol{\phi}) \kappa(\boldsymbol{\phi}+\boldsymbol{\theta})\rangle_{\mathrm{num}}}-1$.

The error bars indicate the $1-\sigma$ deviation across 36 realisations.

- The solid line shows the contribution of the second-order relative to the first-order term computed from the analytical expression including the effective smoothing,

$$
R_{\mu \mu}^{\text {smooth }}=\frac{2 \alpha\left\langle\kappa(\boldsymbol{\phi}) \kappa^{2}(\boldsymbol{\phi}+\boldsymbol{\theta})\right\rangle_{\theta_{\mathrm{S}}}}{\langle\kappa(\boldsymbol{\phi}) \kappa(\boldsymbol{\phi}+\boldsymbol{\theta})\rangle_{\theta_{\mathrm{S}}}}
$$

with $\alpha=2$.

In each case, we use the appropriate reference for $\langle\kappa(\boldsymbol{\phi}) \kappa(\boldsymbol{\phi}+\boldsymbol{\theta})\rangle$, i.e. the numerical measurement in the first and the analytical estimation in the second case. Indeed, the measurement of $\langle\kappa \kappa\rangle$ from the simulation agrees with the analytical estimation within some uncertainty, which is due to numerical effects like the finite number of dark-matter boxes used for simulating the light cone. It introduces a bias into our comparisons which is impossible to separate from the real offset due to all higher-order terms of the Taylor expansion that were not taken into account. The two contributions plotted in Fig. 5 are thus of different nature, but are suitable for a relative comparison.

As the lower panel of the large-scale smoothing simulation shows, the simple $4\langle\kappa \kappa\rangle$ estimate of the magnification misses $20 \%$ of the real amplitude near one arcminute. This discrepancy almost vanishes after adding the contribution of the second-order term, which gives at all scales a final agreement on the per cent level: the additional amplitude reaches $19 \%$ at the smallest scales of the figure, compared to a value of $20 \%$ given by the simulation, and agrees within better than one per cent on larger scales. Therefore, taking into account the $2 \alpha\left\langle\kappa \kappa^{2}\right\rangle$ correction allows the accuracy to be increased by a factor of $\sim 20$ compared to the approximation $4\langle\kappa \kappa\rangle$, in the case of our large-scale smoothing simulation. On the largest scales, between 6 and 30 arcmin, the agreement even improves. Above these scales, the numerical results do not allow any relevant comparison because the number of available 

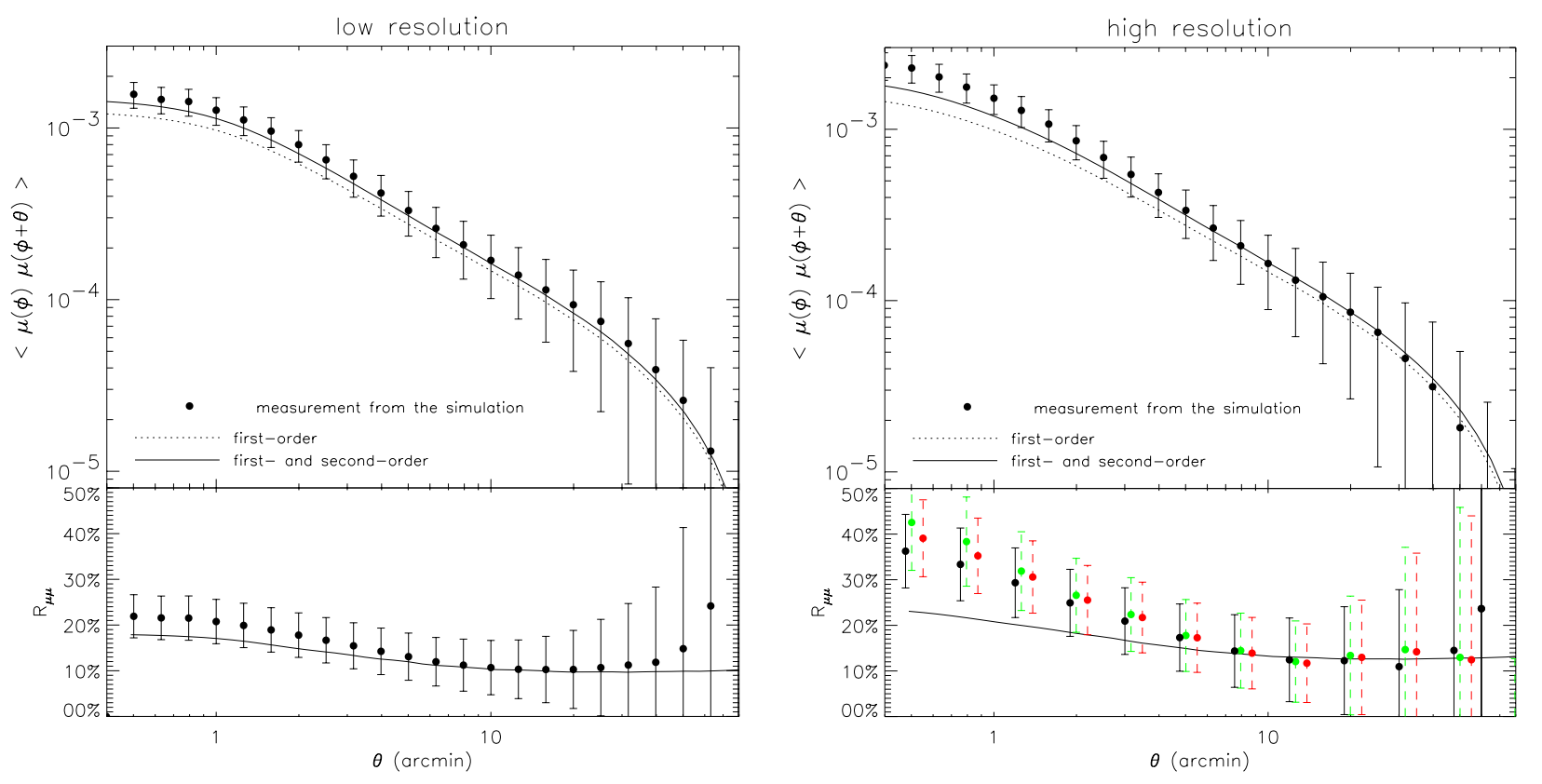

Fig. 5. The upper panel shows a comparison between the magnification autocorrelation measured from the simulation compared to the analytical estimation for a source redshift of unity. The circles show averaged measurements from 36 realisations of the simulation and the corresponding $1-\sigma$ error bars. The dashed line shows the analytical estimation using the approximation $\mu \approx 1+2 \kappa$. The solid line shows the improvement given by the second-order term of the Taylor expansion of $\mu$. The lower panel shows the relative contribution $R_{\mu \mu}$, both measured from the simulation and estimated using expansion terms of $\mu$ up to second order. In the right panel, each angular point shows three different measurements, taking into account the complete magnification maps (solid-line error bars), or maps where pixels were masked where the magnification value exceeds 8 or 4 (dashed-line error bars, from left to right).

independent samplings corresponding to a given separation decreases. On scales below a few arcminutes, the offset between the measured points and the analytical estimate gives the amplitude of all higher-order terms neglected in the Taylor expansion of the magnification. As we can see, their contribution is on the one per cent level for the large-scale smoothing simulation.

The curves shown in the right panel demonstrate how the use of a smaller smoothing scale increases the discrepancy between the analytical and the numerical results. The fraction of non-weakly magnified light rays increases, and caustics appear which give rise to a power-law tail in the magnification probability distribution. We investigate the impact of the rare highly magnified light rays by masking pixels where the simulated magnification exceeds 4 or 8 , and show that caustics have no noticeable effect on the amplitude of the magnification autocorrelation function determined from these simulated data. Note, however, that the impact of the caustics depends on the source redshift. The higher the redshift, the more caustics appear, and the larger is their impact on the correlation amplitude.

Imposing lower masking thresholds removes a significant fraction of the area covered by the simulation, changing the spatial magnification pattern and thus the magnification autocorrelation function. The corresponding measurements are represented by the dashed error bars in the lower right panel of Fig. 5. We note that the error bars of $R_{\mu \mu}^{\text {num }}$ computed with the small-scale smoothing simulation become larger at small scales compared to the lower left panel. This reflects the fact that second-order magnification statistics are ill-defined once caustics appear. In the next section, we will investigate similar smoothing effects on cross-correlations between magnification and dark matter fluctuations. These quantities are not affected by problems of poor definition when the smoothing scale becomes small, and therefore do not show larger error bars at small scales when the smoothing scale decreases.

These comparisons show that the approximation $\mu \approx 1+2 \kappa$ misses a non-negligible part of the total amplitude of weaklensing magnification statistics. The formalism introduced in Sect. 2 allows second-order corrections to be described with or without smoothing of the density field. This provides a better description of the correlation functions, but still gives a lower amplitude than the simulation results. As we noticed, the analytic computation based on the Taylor expansion is sufficiently accurate only in the weak lensing regime. In reality, however, the strong lensing, which can not be taken into account in the analytic formalism, has a significant impact on the magnification correlation especially at small scales as shown in the smallscale smoothing simulation. Therefore, one should carefully take the strong lensing effect into consideration when one interprets the magnification related correlation functions. However, we will see in the next section that counts-in-cells estimators are less affected by the strong lensing than correlation functions and thus enable better comparisons of observations with results from simulations.

\section{Applications to quasar-galaxy correlations}

As a direct application of the formalism introduced previously, we now investigate the effects of second-order terms on a well-known magnification-induced correlation, namely 
the quasar-galaxy cross-correlation (the results can also be applied to galaxy-galaxy correlations induced by magnification; Moessner \& Jain 1998). In order to estimate cosmological parameters from this kind of correlations, we then suggest the use of a more suitable estimator using counts-in-cells rather than two-point correlation functions. It has the advantage of making the observational results more easily reconciled with the ones from numerical simulations.

\subsection{Formalism and correcting terms}

The magnification bias of large-scale structures, combined with galaxy biasing, leads to a cross-correlation of distant quasars with foreground galaxies. The existence of this cross-correlation has firmly been established (e.g. Benítez \& Martínez-González 1995; Williams \& Irwin 1998; Norman \& Impey 1999; Norman \& Williams 2000; Benítez et al. 2001; Norman \& Impey 2001). Ménard \& Bartelmann (2002) showed that the Sloan Digital Sky Survey (York et al. 2000) will allow this correlation function to be measured with a high accuracy. Its amplitude and angular shape contain information on cosmological parameters and the galaxy bias factor. Thus, it is important to accurately describe these magnification-related statistics in order to avoid a biased estimation of cosmological parameters as well as the amplitude of the galaxy bias.

As shown in Bartelmann (1995), the lensing-induced crosscorrelation function between quasars and galaxies can be written as

$$
\begin{aligned}
w_{\mathrm{QG}}(\theta) & \equiv\left\langle\delta_{\mathrm{QSO}}(\boldsymbol{\phi}) \delta_{\mathrm{gal}}(\boldsymbol{\phi}+\boldsymbol{\theta})\right\rangle \\
& =\left\langle\delta \mu^{\alpha-1}(\boldsymbol{\phi}) \delta_{\mathrm{gal}}(\boldsymbol{\phi}+\boldsymbol{\theta})\right\rangle .
\end{aligned}
$$

Using the above formalism, we can expand the effective magnification fluctuation $\delta \mu^{\alpha-1}$ up to second order and find the correcting term:

$w_{\mathrm{QG}}(\theta)=2(\alpha-1)\left[\left\langle\kappa \delta_{\mathrm{gal}}\right\rangle+\alpha\left\langle\kappa^{2} \delta_{\mathrm{gal}}\right\rangle\right]$.

The second term is proportional to $\alpha$ (contrary to the factor $2 \alpha$ in Eq. (5)), since there is only one contribution of the magnification. Therefore, the expected effects will be roughly half of those on the autocorrelation of the effective magnification seen in the previous section. Assuming a linear bias $b$ between galaxies and dark matter, the cross-correlation between $\delta_{\text {gal }}$ and $\kappa^{2}$ can be written as

$$
\begin{aligned}
\left\langle\delta_{\text {gal }}(\boldsymbol{\phi}) \kappa^{2}(\boldsymbol{\phi}+\boldsymbol{\theta})\right\rangle= & b\left\langle\delta_{D M}(\boldsymbol{\phi}) \kappa^{2}(\boldsymbol{\phi}+\boldsymbol{\theta})\right\rangle \\
= & \int \mathrm{d} w \frac{p_{K}^{2}(w) p_{\delta}(w)}{f_{K}^{4}(w)} \int \frac{\mathrm{d}^{2} s_{1}}{(2 \pi)^{2}} \int \frac{\mathrm{d}^{2} s_{2}}{(2 \pi)^{2}} \\
& \times B_{\delta}\left(\frac{s_{1}}{f_{k}(w)}, \frac{s_{2}}{f_{k}(w)}, \frac{-s_{1}-s_{2}}{f_{k}(w)}, w\right) \mathrm{e}^{\mathrm{i} s_{1} \boldsymbol{\theta}},
\end{aligned}
$$

where $p_{\delta}(w)$ is the normalised distance distribution of the galaxies. For this example, we will use

$p_{\delta}(z) \mathrm{d} z=\frac{\beta z^{2}}{z_{0}^{3} \Gamma(3 / \beta)} \exp \left[-\left(\frac{z}{z_{0}}\right)^{\beta}\right] \mathrm{d} z$,

with $\beta=1.5$ and $z_{0}=0.3$.

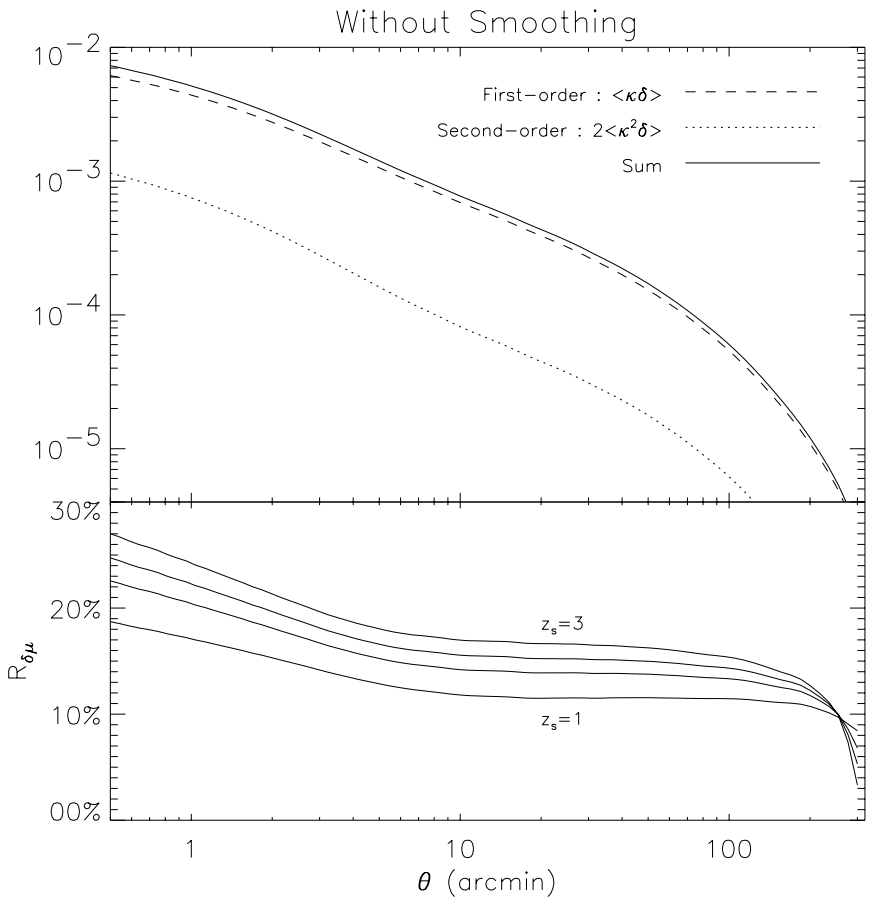

Fig. 6. The upper panel shows the amplitude of the normalised quasargalaxy correlation $w_{\mathrm{QG}} / 2(\alpha-1)$ as a function of angular separation. We show the first two terms of the Taylor expansion of this correlation, namely $\langle\delta(\boldsymbol{\phi}) \kappa(\boldsymbol{\phi}+\boldsymbol{\theta})\rangle$ (dashed line) and $2\left\langle\delta(\boldsymbol{\phi}) \kappa^{2}(\boldsymbol{\phi}+\boldsymbol{\theta})\right\rangle$ (dotted line), using a source redshift of unity. The sum of these two terms is shown as the solid line. The lower panel details the relative contribution $R_{\delta \mu}$ and of the second-order term for different source redshifts, namely $z=1,1.5,2$ and 3 from bottom to top.

The results are shown in Fig. 6. As we can see, previous estimates using the approximation $\mu \approx 2 \kappa$ missed approximately $15 \%$ of the amplitude on small scales for quasars at redshift unity. Using quasars at redshift 2, these effects reach up to $25 \%$. These offsets, which are only lower limits, would lead to biased estimates of $\Omega_{0}$ or $b$, for example.

As for the magnification autocorrelation, we can compare our theoretical estimates against numerical estimations. We can first introduce a coefficient $R_{\delta \mu}$ describing the accuracy of our second-order correction:

$R_{\delta \mu}(\theta)=\frac{\alpha\left\langle\delta(\phi) \kappa^{2}(\boldsymbol{\phi}+\boldsymbol{\theta})\right\rangle}{\langle\delta(\boldsymbol{\phi}) \kappa(\boldsymbol{\phi}+\boldsymbol{\theta})\rangle}$

We plot the results in Fig. 7. Note that contrary to the magnification autocorrelation, this quantity does not suffer from poor definition, even without smoothing. The difference can be seen by the same size of the error bars between the two simulation results at small scales, whereas they were larger in the case of $\langle\mu \mu\rangle$ for the small-scale smoothing simulation (Fig. 5). The results for $R_{\delta \mu}$ are very similar those obtained for $R_{\mu \mu}$ : for the large-scale smoothing ray-tracing we find very good agreement which reaches the one percent level on small scales. However, when the smoothing length decreases, we see from the smallscale smoothing outputs that we are missing a part of the total amplitude on small scales, which shows that higher-order terms play a non negligible role on those scales. 


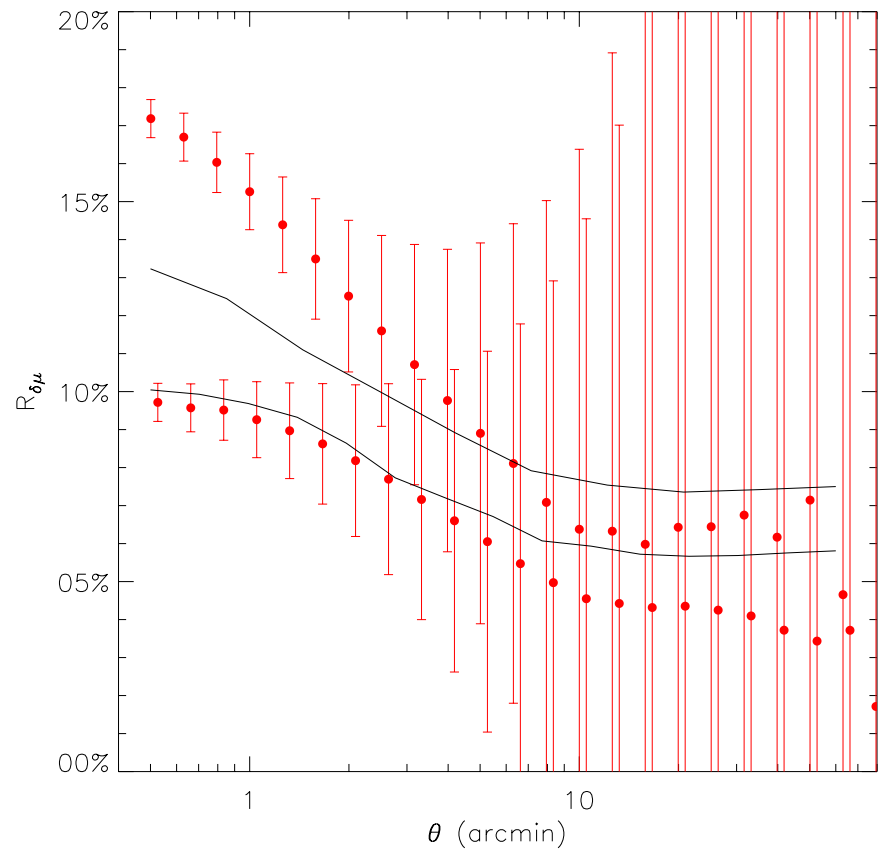

Fig. 7. Comparison of $R_{\delta \mu}$ given by the theoretical calculation and measured from the numerical simulation. The results are shown for the large- and small-scale smoothing simulations from bottom to top. They show that a second-order description of the $\langle\mu \delta\rangle$ crosscorrelation gives good results when the smoothing is large, but misses some part of the total amplitude in the case of our small-scale smoothing ray-tracing.

\subsection{Using count-in-cells estimators}

For precisely estimating cosmological parameters as well as the amplitude of the galaxy bias, it is necessary to employ theoretical magnification statistics that closely describe the observables. However, we have seen in Sect. 3 that analytical estimates as well as numerical simulations have intrinsic limitations and prevent us from accurately describing usual $n$-point correlation functions related to magnification statistics.

Besides, it is possible to focus on another estimator closely related to correlation functions, namely a count-in-cells estimator, which naturally smoothes effects originating from the density field and can thus more easily be reconciled with numerical simulations. So far, quasar-galaxy or galaxy-galaxy correlations have been quantified measuring the excess of background-foreground pairs at a given angular separation. Instead, we can correlate the amplitude of the background and foreground fluctuations, both measured inside a given aperture. We will therefore introduce a count-in-cells estimator,

$$
\begin{aligned}
\bar{w}_{\mathrm{QG}}(\theta)= & \left\langle\delta_{\mathrm{QSO}}(\boldsymbol{\phi}) \delta_{\mathrm{gal}}(\boldsymbol{\phi})\right\rangle_{\theta} \\
& \left\langle\delta \mu^{\alpha-1}(\boldsymbol{\phi}) \delta_{\mathrm{gal}}(\boldsymbol{\phi})\right\rangle_{\theta},
\end{aligned}
$$

where the subscript $\theta$ indicates averaging of $\delta_{\mathrm{QSO}}(\phi)$ and $\delta_{\text {gal }}(\phi)$ inside a cell of radius $\theta$. In practice, this estimator is intended to be applied to galaxy-galaxy rather than to quasar-galaxy correlations, since the average angular separation between bright distant quasars is of order one degree for current surveys, thus averaging the source counts inside cells with radii of several arcminutes will not be relevant. Using galaxies as background sources, this limitation occurs at much smaller scales.

Using a first-order Taylor expansion for the magnification, the new estimator $\bar{w}_{\mathrm{QG}}(\theta)$ can be written

$$
\begin{aligned}
\frac{\bar{w}_{\mathrm{QG}}(\theta)}{2(\alpha-1) b}= & \int \mathrm{d} w \frac{p_{\kappa}(w) p_{\delta}(w)}{f_{K}^{2}(w)} \int \frac{s \mathrm{~d} s}{2 \pi} \\
& \times P\left(\frac{s}{f_{k}(w)}, w\right)|I(s \theta)|^{2},
\end{aligned}
$$

where $I(x)=2 \frac{J_{1}(x)}{x}$. This expression differs from the 2-point correlation function (9) by its Fourier-space filtering of the power spectrum. The additional smoothing wipes out the power on scales smaller than the physical scale corresponding to the angular smoothing scale $\theta$. For any observational result to be compared to a numerical simulation, $\theta$ and the smoothing scale used in the simulation will have to be carefully adapted to each other and to the redshift distribution of the foreground galaxy distribution.

In practice, masking always makes correlation functions easier to measure than counts-in-cells. However, in a large survey with short exposures like the SDSS, masking is not a real issue to measure counts-in-cells since unusable regions are quite rare and their area is small compared to the total survey size. This is different for cosmic shear surveys for which images are deeper and saturation occurs more frequently.

Note that gravitational lensing by the foreground galaxies themselves is entirely irrelevant here. The angular scale on which galaxies act as efficient lenses is on the order of one arc second and below, much smaller than the angular scales we are concerned with. Moreover, the probability for a quasar to be strongly lensed by a galaxy is well below one per cent. Bartelmann \& Schneider (1991) demonstrated this point explicitly by including galaxies into their numerical simulations and showing they had no noticeable effect.

\section{Conclusion}

As surveys mapping the large-scale structure of the Universe become wider and deeper, measuring cosmological parameters as well as the galaxy bias with cosmic magnification will become increasingly efficient and reliable. Therefore, an accurate theoretical quantification of magnification statistics becomes increasingly important.

Previous estimates of cosmic magnification relied on the assumption that the magnification deviates sufficiently little from unity that it can be accurately approximated by its firstorder Taylor expansion about unity, i.e. $\mu \approx 1+2 \kappa$. In this paper, we have tested the validity of this assumption in the framework of magnification statistics, by investigating the second-order terms in the Taylor expansion of $\mu$. We have shown that:

- Second-order terms can be related to the cross-correlation between $\kappa$ and $\kappa^{2}$;

- their importance increases as the number-count function of the background sources steepens, i.e. as $\alpha$ increases; 
- their amplitude is not negligible: for the magnification autocorrelation, their contribution is typically on the order of $30 \%-50 \%$ at scales below one degree. Therefore, previous estimates of cosmic magnification were systematically low.

For testing our theoretical calculations, we have compared our results to magnification statistics found in numerical simulations by performing ray-tracing experiments in a very large $N$-body simulation. We have first checked the validity of our formalism describing the correlation $\left\langle\kappa \kappa^{2}\right\rangle$, and demonstrated the importance of including an effective smoothing into the analytical calculations. Indeed, $\mu$ is nonlinear in the density field and the amplitude of magnification statistics measured from numerical simulations depends therefore on the available resolution.

Using a simulation with an effective smoothing scale of 0.8 arcmin, we found that our second-order formalism is accurate to the percent level for describing magnification autocorrelations. Compared to previous estimates, this improves the accuracy by a factor of $\approx 20$. For smaller effective smoothing scales, the contribution of third- and higher-order terms becomes important on scales below a few arcminutes.

Finally we have applied our formalism to observed correlations, like quasar-galaxy and galaxy-galaxy correlations due to lensing. We have shown that second-order corrections increase their amplitude by $15 \%$ to $25 \%$ on scales below one degree. These correlations are valuable tools to probe cosmological parameters as well as the galaxy bias. However, even including our correcting terms, analytical or numerical estimates of magnification statistics can only provide lower bounds to the real amplitude of the correlation functions in the weak-lensing regime. Thus, we propose using count-in-cells estimators rather than correlation functions since the intrinsic smoothing in determining counts-in-cells allows the observational results to be more directly related to those obtained in numerical simulations.

Thus, some care is required in using cosmic magnification as described by a Taylor expansion for constraining cosmological parameters, especially for interpreting measurements on small angular scales. Therefore, describing magnification statistics using the halo-model formalism will be of great interest in order to achieve a precise and direct description of observational quantities.

Acknowledgements. We thank Francis Bernardeau and Stéphane Colombi for helpful discussions. This work was supported in part by the TMR Network "Gravitational Lensing: New Constraints on Cosmology and the Distribution of Dark Matter" of the EC under contract No. ERBFMRX-CT97-0172.

\section{Appendix A: Bispectrum and non-linear evolution}

The bispectrum can be estimated using second-order perturbation theory. Indeed, an expansion of the density field to second nonlinear order as

$\delta(\boldsymbol{x}) \approx \delta^{(1)}(\boldsymbol{x})+\delta^{(2)}(\boldsymbol{x})$, where $\delta^{(2)}$ is of order $\left(\delta^{(1)}\right)^{2}$ and represents departures from Gaussian behaviour, yields the bispectrum

$$
\begin{aligned}
\left\langle\delta_{1} \delta_{2} \delta_{3}\right\rangle \simeq & \left\langle\delta_{1}^{(1)} \delta_{2}^{(1)} \delta_{3}^{(1)}\right\rangle+\left\langle\delta_{1}^{(1)} \delta_{2}^{(1)} \delta_{3}^{(2)}\right\rangle \\
& + \text { cyclic terms }(231,312) .
\end{aligned}
$$

The first term in Eq. (A.2) vanishes because the density fluctuation field is Gaussian to first order, hence the third moment of $\delta^{(1)}$ is zero. Thus, the leading term in Eq. (A.2) is of the order of $\left\langle\delta_{1}^{(1)} \delta_{2}^{(1)} \delta_{3}^{(2)}\right\rangle$ and can be quantified using second-order perturbation theory.

The bispectrum $B_{\delta}\left(\boldsymbol{k}_{1}, \boldsymbol{k}_{2}, \boldsymbol{k}_{3}\right)$ is defined only for closed triangles formed by the wave vectors $\boldsymbol{k}_{1}, \boldsymbol{k}_{2}, \boldsymbol{k}_{3}$. It can be expressed as a function of the second-order kernel $F\left(\boldsymbol{k}_{1}, \boldsymbol{k}_{2}\right)$ and the power spectrum

$$
\begin{aligned}
B_{\delta}\left(\boldsymbol{k}_{1}, \boldsymbol{k}_{2}, \boldsymbol{k}_{3}\right)= & 2 F\left(\boldsymbol{k}_{1}, \boldsymbol{k}_{2}\right) P\left(k_{1}\right) P\left(k_{2}\right) \\
& +2 F\left(\boldsymbol{k}_{2}, \boldsymbol{k}_{3}\right) P\left(k_{2}\right) P\left(k_{3}\right) \\
& +2 F\left(\boldsymbol{k}_{1}, \boldsymbol{k}_{3}\right) P\left(k_{1}\right) P\left(k_{3}\right) .
\end{aligned}
$$

For describing the bispectrum on all scales, we use the fitting formula derived by Scoccimarro \& Couchman (2000) for the non-linear evolution of the bispectrum in numerical simulations of CDM models, extending previous work for scale-free initial conditions. In that case, we have

$$
\begin{aligned}
F\left(\boldsymbol{k}_{1}, \boldsymbol{k}_{2}\right)= & \frac{5}{7} a\left(n, k_{1}\right) a\left(n, k_{2}\right) \\
& +\frac{1}{2} \frac{\boldsymbol{k}_{1} \cdot \boldsymbol{k}_{2}}{k_{1} k_{2}}\left(\frac{k_{1}}{k_{2}}+\frac{k_{2}}{k_{1}}\right) b\left(n, k_{1}\right) b\left(n, k_{2}\right) \\
& +\frac{2}{7}\left(\frac{\boldsymbol{k}_{1} \cdot \boldsymbol{k}_{2}}{k_{1} k_{2}}\right)^{2} c\left(n, k_{1}\right) c\left(n, k_{2}\right),
\end{aligned}
$$

with

$a(n, k)=\frac{1+\sigma_{8}^{-0.2}(z)\left[0.7 Q_{3}(n)\right]^{1 / 2}(q / 4)^{n+3.5}}{1+(q / 4)^{n+3.5}}$

$b(n, k)=\frac{1+0.4(n+3) q^{n+3}}{1+q^{n+3.5}}$

$c(n, k)=\frac{1+4.5 /\left[1.5+(n+3)^{4}\right](2 q)^{n+3}}{1+(2 q)^{n+3.5}}$,

and $q \equiv k / k_{\mathrm{NL}}(z)$, where $4 \pi k_{\mathrm{NL}}^{3} P_{L}\left(k_{\mathrm{NL}}\right)=1$, and $P_{\mathrm{L}}(k)$ is the linear power spectrum at the desired redshift. The effective spectral index is taken from the linear power spectrum as well. The function $Q_{3}(n)$ is given by

$Q_{3}(n)=\frac{\left(4-2^{n}\right)}{\left(1+2^{n+1}\right)}$.

For more detail, see Scoccimarro \& Couchman (2000).

\section{References}

Bacon, D., Massey, R., Réfrégier, A., \& Ellis, R. 2002, MNRAS, submitted [astro-ph/0203134]

Bartelmann, M. 1995, A\&A, 298, 661

Benítez, N., \& Martínez-González, E. 1995, ApJ, 448, L89 
Benítez, N., Sanz, J. L., \& Martínez-González, E. 2001, MNRAS 320, 241

Norman, D. J., \& Impey, C. D. 1999, AJ, 118, 613

Norman, D. J., \& Williams, L. L. R. 2000, AJ, 119, 2060

Blandford, R. D., Saust, A. B., Brainerd, T. G., \& Villumsen, J. V. 1991, MNRAS, 251, 600

Norman, D. J., \& Impey, C. D. 2001, ApJ, 552, 473

Peacock, J. A., \& Dodds, S. J. 1996, MNRAS, 280, L19

Couchman, H. M. P., Thomas, P. A., \& Pearce, F. R. 1995, ApJ, 452, 797

Pearce, F. R., \& Couchman, H. M. P. 1997, NewA, 2, 411

Pei, Y. C. 1995, ApJ, 438, 623

Guimarães, A. C. C., van de Bruck, C., \& Brandenberger, R. H. 2001, MNRAS, 325, 278

Hamana, T., Martel, H., \& Futamase, T. 2000, ApJ, 529, 56

Hamana, T., \& Mellier, Y. 2001, MNRAS, 169, 176

Hamana, T., Yoshida, N., \& Suto, Y. 2002, ApJ, 568, 455

Haemmerle, H., J.-M. Miralles, J.-M., Schneider, P., et al. 2002, A\&A, 385,743

Hockney, R. W., \& Eastwood, J. W. 1988, Computer Simulation Using Particles (Bristol: Adam Hilger)

Hoekstra, H., Yee, H. K. C., Gladders, M. D., et al. 2002, ApJ, 572, 55

Jain, B., Seljak, U., \& White, S. D. M. 2000, ApJ, 530, 547

Kaiser, N., Wilson, G., \& Luppino, G. A. 2000, ApJL, submitted [astro-ph/0003338]

Maoli, R., Van Waerbeke, L., Mellier, Y., et al. 2001, A\&A, 368, 766

Ménard, B., \& Bartelmann, M. 2002, A\&A, 386, 784

Ménard, B., Bartelmann, M., \& Mellier, Y. 2002, A\&A, submitted [astro-ph/0208361]

Moessner, R., \& Jain, B. 1998, MNRAS, 294, L18

Rhodes, J., Réfrégier, A., \& Groth, E. 2001, ApJ, 552, L85

Schneider, P., Ehlers, J., \& Falco, E. E. 1992, Gravitational Lenses (Heidelberg: Springer Verlag)

Scoccimarro, R., \& Couchman, H. M. P. 2001, MNRAS, 325, 1312

Seljak, U., \& Zaldarriaga, M. 1996, ApJ, 469, 437

Van Waerbeke, L., Mellier, Y., Erben, T., et al. 2000, A\&A, 358, 30

Van Waerbeke, L., Mellier, Y., Radovich, M., et al. 2001a, A\&A, 374, 757

Van Waerbeke, L., Hamana, T., Scoccimarro, R., Colombi, S., \& Bernardeau, F. 2001b, MNRAS 322, 918

Van Waerbeke, L., Mellier, Y., Pelló, R., et al. 2002, A\&A, 393, 369

White, M., \& Hu, W. 2000, ApJ, 537, 1

Williams, L. L. R., \& Irwin, M. 1998, MNRAS, 298, 378

Wittman, D. M., Tyson, J. A., Kirkman, D., Dell'Antonio, I., \& Bernstein, G. 2000, Nature, 405, 143

Yoshida, N. Sheth, R. K., \& Diaferio, A. 2001, MNRAS, 328, 669

York, D. G., Adelman, J., Anderson, J. E., Anderson, S. F., et al. 2000, AJ, 120, 1607 